SUBROUTINE Homogeneous_Fluence_Fluorescence_Infinite

$+($

+mua, ! Absorption coefficient of the medium (mm-1): mua(1) at the excitation wavelength, mua(2) at the emission wavelength

+musp, ! Reduced scattering coefficient of the medium (mm-1): musp(1) at the excitation wavelength, musp(2) at the emission wavelength

$+\mathrm{s}, \quad$ ! Thickness of the slab (mm)

$+\mathrm{t}, \quad$ ! Time (ps)

$+\mathrm{xs}, \quad$ !x position of the source $(\mathrm{mm})$

+ys, ! ! y position of the source $(\mathrm{mm})$

+zs, $\quad$ ! z position of the source $(\mathrm{mm})$

$+\mathrm{xr}, \quad$ !x position of the receiver $(\mathrm{mm})$

$+\mathrm{yr}, \quad$ ! y position of the receiver $(\mathrm{mm})$

$+\mathrm{zr}, \quad$ ! $\mathrm{z}$ position of the receiver $(\mathrm{mm})$

$+\mathrm{xp}, \quad$ ! x position of the fluorophore $(\mathrm{mm})$

+yp, ! y position of the fluorophore $(\mathrm{mm})$

+zp, ! $\quad$ z position of the fluorophore $(\mathrm{mm})$

$+\mathrm{n} 0, \quad$ ! Refractive index of the medium: n0(1) at the excitation wavelength, $\mathrm{n} 0(2)$ at the emission wavelength

+AA, ! Coefficient for the extrapolated boundary condition: AA(1) at the excitation wavelength, AA(2) at the emission wavelength

+fluence, ! Name of the subroutine to be used to evaluate the TR/CW Fluence (mm-2 ps-1)/(mm-2) for the infinite medium

+tau, ! Fluorescent lifetime of the fluorophore (ps)

+fluore ! Output: TR/CW Fluence Fluorescence (mm-2 ps-1)/(mm-2) for the infinite medium

$+)$

* This subroutine calculates the fluorescence fluence of Eq. (11) and of Eq. (12) for the infinite medium

* IF $\mathrm{t}>=0$ the TR fluorescence is calculated

* The subroutine calculates numerically the TR fluorescence fluence for the homogeneous infinite medium

* (mm-2 ps-1) emitted for a unitary quantum yield (quantum efficiency) using Equation (11) of the paper.

* The calculation is carried out assuming a unitary volume for the fluorophore and a unitary

* absorption coefficient of the fluorophore at the excitation wavelength.

* For obtaining the actual TR fluorescence signal the calculated quantity fluore

* must be multiplied by the quantum efficieny of the fluorophore,

* by the volume occupied by the fluorophore (Unit $\mathrm{mm}^{\wedge} 3$ ), and

* by the absorption coefficient of the fluorophore at the excitation wavelength (Unit $\mathrm{mm}^{\wedge}-1$ ).

* The names of the subroutines that can be used to calculate the fluence of the infinite medium are:

* DE_Fluence_Infinite_TR.for: TR Solution of the Diffusion Equation

* RTE_Fluence_Infinite_TR.for: TR Solution of the Radiative Transfer Equation

* TE_Fluence_Infinite_TR.for: TR Solution of the Telegrapher Equation

* For the above subroutines see the software enclosed with Ref. 21 of the paper

* nd is the parameter used to evaluate numerically the convolution integral of Eq. (11)

* of the paper (number of temporal intervals):

* The time interval $[0, \mathrm{t}]$ is divided into nd steps.

$*$

* Note that the following arguments: s, AA, and n0 are not used.

* IF $\mathrm{t}<0$ the CW fluorescence is calculated 
* The subroutine calculates numerically the CW fluorescence fluence for the homogeneous infinite medium

* (mm-2) emitted for a unitary quantum yield (quantum efficiency) using Equation (12) of the paper.

* The calculation is carried out assuming a unitary volume for the fluorophore and a unitary

* absorption coefficient of the fluorophore at the excitation wavelength.

* For obtaining the actual CW fluorescence signal the calculated quantity fluore

* must be multiplied by the quantum efficieny of the fluorophore,

* by the volume occupied by the fluorophore (Unit $\mathrm{mm}^{\wedge} 3$ ), and

* by the absorption coefficient of the fluorophore at the excitation wavelength (Unit $\mathrm{mm}^{\wedge}-1$ ).

* The names of the subroutines that can be used to calculate the fluence of the infinite medium are:

* DE_Fluence_Infinite_CW.for: CW Solution of the diffusion equation

* IDE_Fluence_Infinite_CW.for: CW improved solution of the DE (IDE) for isotropic scattering proposed by

* Graff and Rinzema (2001).

* Note that the following arguments: s, AA, n0, and t are not used.

\section{IMPLICIT NONE}

EXTERNAL fluence

INTEGER, PARAMETER :: nd = 200

REAL*8 mua(2),musp(2),s,t,xs,ys,zs,xr,yr,zr

REAL*8 xp,yp,zp,n0(2),AA(2),tau

REAL*8 fluore

REAL*8 f1CW,f2CW

REAL*8 f1(nd),g1(nd),tf1(nd),tg1

REAL*8 f2(nd),g2(nd),tf2(nd),tg2

\section{INTEGER i,j}

fluore $=0 . \mathrm{d} 0$

IF (t .lt. 0.d0) THEN

CALL fluence(

+ mua(1),musp(1),s,10.d0,xs,ys,zs,xp,yp,zp,n0(1),

$+\mathrm{AA}(1), \mathrm{f} 1 \mathrm{CW})$

CALL fluence(

+ mua(2),musp(2),s,10.d0,xp,yp,zp,xr,yr,zr,n0(2),

$+\mathrm{AA}(2), \mathrm{f} 2 \mathrm{CW})$

fluore $=\mathrm{f} 1 \mathrm{CW} * \mathrm{f} 2 \mathrm{CW}$

RETURN

END IF

IF (t .eq. 0.d0) RETURN

DO $\mathrm{i}=1$, nd

tf1(i)=t/DBLE(nd-1)*DBLE(i-1) 
$\operatorname{tg} 1=\mathrm{t}-\mathrm{tf} 1(\mathrm{i})$

IF(tau/DBLE(nd)*20.D0.lt.tf1(i)/DBLE(nd-1))THEN

DO $\mathrm{j}=1$,nd

tf2(j)=tf1(i)-tau*DBLE(nd-j)/DBLE(nd)*20.D0

$\operatorname{tg} 2=\operatorname{tau} *$ DBLE(nd-j)/DBLE(nd)*20.D0

CALL fluence(

$+\quad$ mua(1),musp(1),s,tf2(j),xs,ys,zs,xp,yp,zp,n0(1),

$+\quad \mathrm{AA}(1), \mathrm{f} 2(\mathrm{j}))$

$\operatorname{tf} 2(j)=-\operatorname{tg} 2$

g2(j) $=$ dexp $(-\operatorname{tg} 2 / \operatorname{tau}) / \operatorname{tau}$

END DO

ELSE

DO $j=1$,nd

$\mathrm{tf2}(\mathrm{j})=\mathrm{tf} 1(\mathrm{i}) / \mathrm{DBLE}(\mathrm{nd}-1) * \operatorname{DBLE}(\mathrm{j}-1)$

$\operatorname{tg} 2=t f 1(i)-t f 2(j)$

CALL fluence(

$+\quad \operatorname{mua}(1), m u s p(1), s, t f 2(j), x s, y s, z s, x p, y p, z p, n 0(1)$,

$+\quad \mathrm{AA}(1), \mathrm{f} 2(\mathrm{j}))$

$g 2(j)=\operatorname{dexp}(-\operatorname{tg} 2 / \operatorname{tau}) / \operatorname{tau}$

END DO

END IF

CALL TRAP(tf2,f2*g2,nd,f1(i)) ! Convolution with the exponential decay of the fluorophore

CALL fluence(

+ mua(2),musp(2),s,tg1,xp,yp,zp,xr,yr,zr,n0(2),

+ AA(2),g1(i))

END DO

CALL TRAP(tf1,f1*g1,nd,fluore)

RETURN

END 


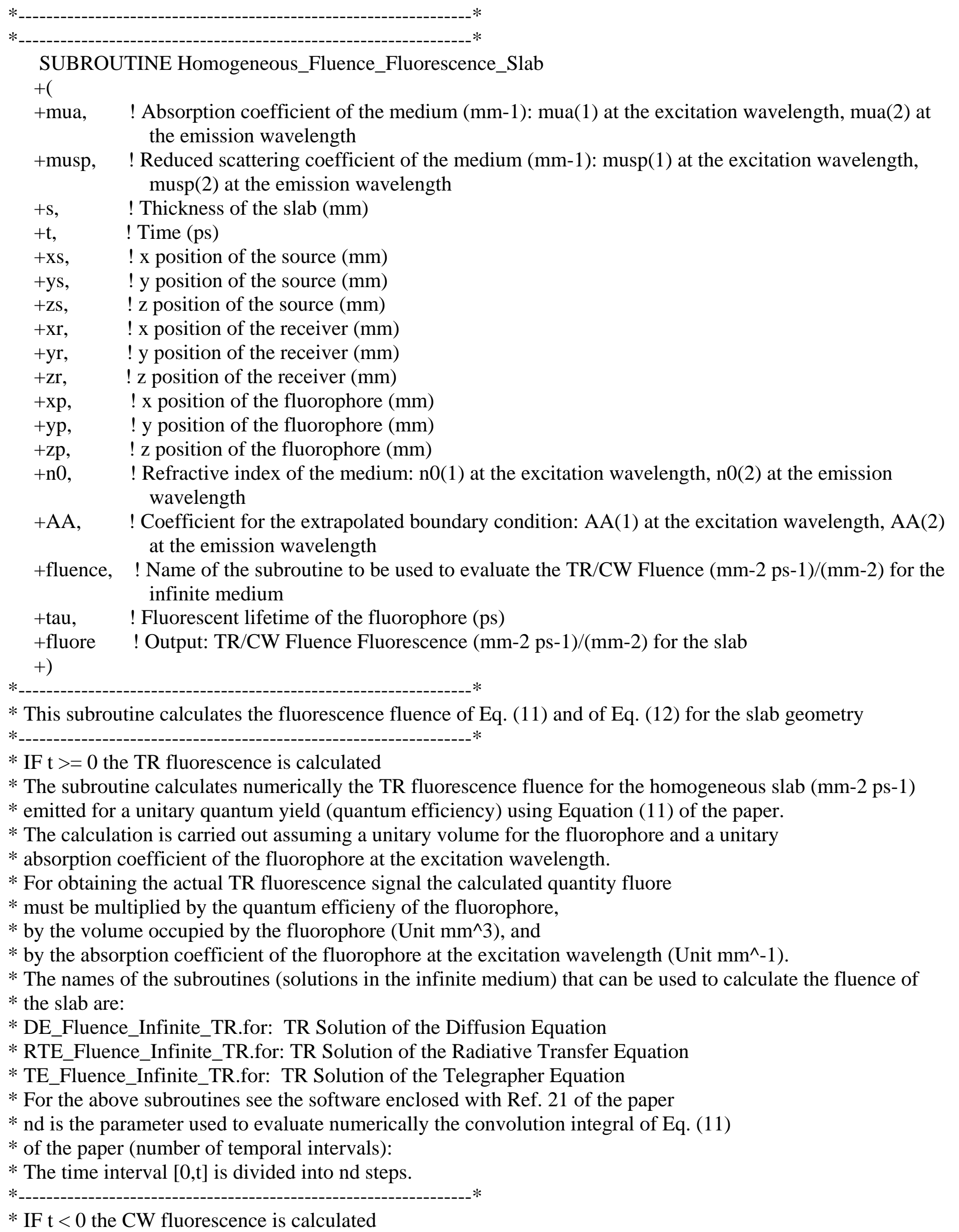


* The subroutine calculates numerically the CW fluorescence fluence for the homogeneous slab (mm-2)

* emitted for a unitary quantum yield (quantum efficiency) using Equation (12) of the paper.

* The calculation is carried out assuming a unitary volume for the fluorophore and a unitary

* absorption coefficient of the fluorophore at the excitation wavelength.

* For obtaining the actual CW fluorescence signal the calculated quantity fluore

* must be multiplied by the quantum efficieny of the fluorophore,

* by the volume occupied by the fluorophore (mm-1), and

* by the absorption coefficient of the fluorophore at the excitation wavelength (Unit $\mathrm{mm}^{\wedge} \mathrm{-}-1$ ).

* The names of the subroutines (solutions in the infinite medium) that can be used to calculate the fluence of

* the slab are:

* DE_Fluence_Infinite_CW.for: CW Solution of the Diffusion Equation

* IDE_Fluence_Infinite_CW.for: CW improved solution of the DE (IDE) for isotropic scattering proposed by

* Graff and Rinzema (2001)

$*$

* Note that the following arguments: $\mathrm{n} 0$ and $\mathrm{t}$ are not used.

IMPLICIT NONE

EXTERNAL fluence

INTEGER, PARAMETER :: nd = 400

REAL*8 mua(2),musp(2),s,t,xs,ys,zs,xr,yr,zr

REAL*8 xp,yp,zp,n0(2),AA(2),tau

REAL*8 fluore

REAL*8 f1CW,f2CW

REAL*8 f1(nd),g1(nd),tf1(nd),tg1

REAL*8 f2(nd),g2(nd),tf2(nd),tg2

\section{INTEGER i,j}

fluore $=0 . \mathrm{d} 0$

IF (t .lt. 0.d0) THEN

CALL Homogeneous_Fluence_Slab(

+ mua(1),musp(1),s,10.d0,xs,ys,zs,xp,yp,zp,n0(1),

+ AA(1),fluence,f1CW)

CALL Homogeneous_Fluence_Slab(

$+\operatorname{mua}(2), \operatorname{musp}(2), \mathrm{s}, 10 . \mathrm{d} 0, \mathrm{xp}, \mathrm{yp}, \mathrm{zp}, \mathrm{xr}, \mathrm{yr}, \mathrm{zr}, \mathrm{n} 0(2)$,

$+\mathrm{AA}(2)$,fluence,f2CW)

fluore $=\mathrm{f} 1 \mathrm{CW} * \mathrm{f} 2 \mathrm{CW}$

RETURN

END IF

IF (t .eq. 0.d0) RETURN

DO i=1,nd 
$\mathrm{tf1}(\mathrm{i})=\mathrm{t} / \mathrm{DBLE}(\mathrm{nd}-1) * \operatorname{DBLE}(\mathrm{i}-1)$

$\operatorname{tg} 1=t-t f 1(i)$

IF(tau/DBLE(nd)*20.D0.lt.tf1(i)/DBLE(nd-1))THEN

DO $\mathrm{j}=1$,nd

tf2(j)=tf1(i)-tau*DBLE(nd-j)/DBLE(nd)*20.D0

$\operatorname{tg} 2=\operatorname{tau} *$ DBLE(nd-j)/DBLE(nd)*20.D0

CALL Homogeneous_Fluence_Slab(

$+\quad \operatorname{mua(1),musp(1),s,tf2(j),xs,ys,zs,xp,yp,zp,n0(1),~}$

$+\quad$ AA(1),fluence,f2(j))

$\operatorname{tf} 2(j)=-\operatorname{tg} 2$

g2(j) $=\operatorname{dexp}(-\operatorname{tg} 2 / \operatorname{tau}) / \operatorname{tau}$

\section{END DO}

\section{ELSE}

DO $j=1$,nd

$\mathrm{tf2}(\mathrm{j})=\mathrm{tf1}(\mathrm{i}) / \mathrm{DBLE}(\mathrm{nd}-1) * \operatorname{DBLE}(\mathrm{j}-1)$

$\operatorname{tg} 2=\operatorname{tf} 1(i)-\operatorname{tf} 2(j)$

CALL Homogeneous_Fluence_Slab(

$+\quad \operatorname{mua}(1), \operatorname{musp}(1), \mathrm{s}, \mathrm{tf} 2(\mathrm{j}), \mathrm{xs}, \mathrm{ys}, \mathrm{zs}, \mathrm{xp}, \mathrm{yp}, \mathrm{zp}, \mathrm{n} 0(1)$,

$+\quad$ AA(1),fluence,f2(j))

$g 2(j)=\operatorname{dexp}(-\operatorname{tg} 2 / \operatorname{tau}) / \operatorname{tau}$

\section{END DO}

\section{END IF}

CALL TRAP(tf2,f2*g2,nd,f1(i)) ! Convolution with the exponential decay of the fluorophore

CALL Homogeneous_Fluence_Slab(

+ mua(2),musp(2),s,tg1,xp,yp,zp,xr,yr,zr,n0(2),

+ AA(2),fluence,g1(i))

END DO

CALL TRAP(tf1,f1*g1,nd,fluore)

RETURN 


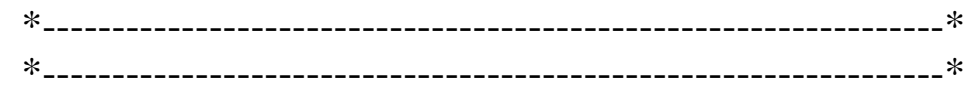

SUBROUTINE DE_Fluence_Infinite_CW

$+($

+mua, ! Absorption coefficient of the medium (mm-1)

+musp, ! Reduced scattering coefficient of the medium (mm-1)

$+\mathrm{s}, \quad$ ! Thickness of the slab (mm)

$+\mathrm{t}, \quad$ ! Time (ps)

+xs, ! ! position of the source $(\mathrm{mm})$

+ys, !y position of the source $(\mathrm{mm})$

+zs, ! z position of the source (mm)

$+\mathrm{xr}, \quad ! \mathrm{x}$ position of the receiver $(\mathrm{mm})$

$+\mathrm{yr}, \quad$ !y position of the receiver $(\mathrm{mm})$

$+\mathrm{zr}, \quad$ ! $\mathrm{z}$ position of the receiver $(\mathrm{mm})$

$+\mathrm{n} 0, \quad$ ! Refractive index of the medium

+AA, ! Coefficient for the extrapolated boundary condition

+fluence ! Output: DE CW Fluence per unit emitted energy (mm-2) in the infinite medium $+)$

* This subroutine calculates the CW fluence obtained from the solution the diffusion equation (DE) for the * infinite medium

* Fluence : Equation 4.6 of Ref. 21, i.e. of F. Martelli, S. Del Bianco, A. Ismaelli, and G. Zaccanti Light

* Propagation through Biological Tissue and Other Diffusive Media: Theory, Solutions, and Software, SPIE

* PRESS, Bellingham (2010).

$*$

$*$

* Note that the following arguments: t, s, n0, AA are not used.

\section{$*$}

IMPLICIT NONE

REAL*8 mua,musp,s,t,xs,ys,zs, xr,yr,zr,n0,AA

$\mathrm{REAL} * 8$ fluence

REAL*8 pi,D,r,mueff,sig

fluence $=0 . \mathrm{d} 0$

$\mathrm{pi}=2 . \mathrm{d} 0 *$ dasin $(1 . \mathrm{d} 0)$

$\mathrm{D}=1 . \mathrm{d} 0 /(3 . \mathrm{d} 0 *$ musp)

$\mathrm{r}=\mathrm{dsqrt}((\mathrm{xr}-\mathrm{xs}) * * 2 . \mathrm{d} 0+(\mathrm{yr}-\mathrm{ys}) * * 2 . \mathrm{d} 0+(\mathrm{zr}-\mathrm{zs}) * * 2 . \mathrm{d} 0)$

mueff $=$ dsqrt $(3 . d 0 *$ musp*mua $)$

sig=dsqrt(mua/D)

fluence $=3 . \mathrm{d} 0 *$ musp/(4.d0* $\left.\mathrm{pi}{ }^{*} \mathrm{r}\right) * \mathrm{dexp}(-\mathrm{r} *$ mueff $)$

RETURN

END 


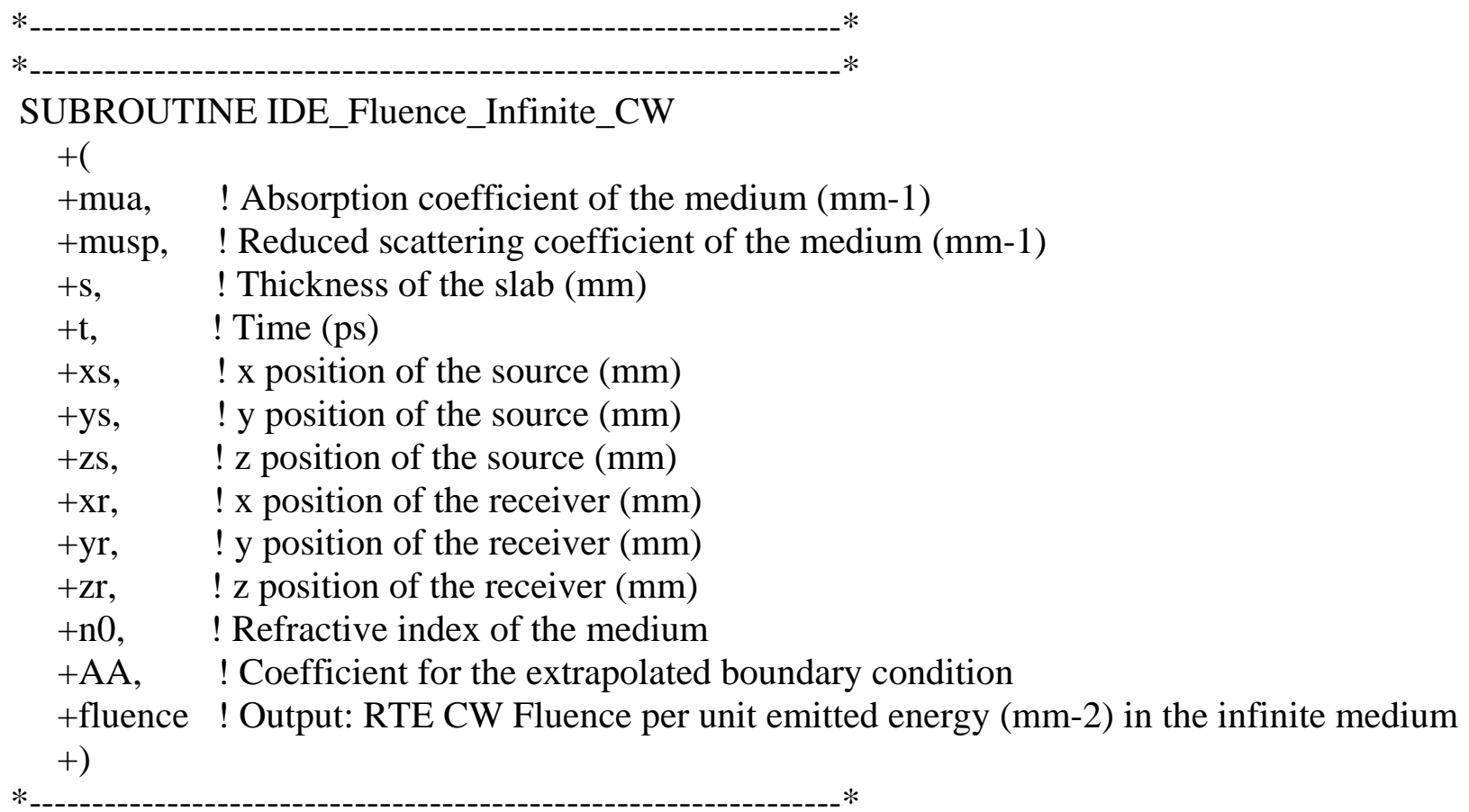

* This subroutine calculates the CW fluence for the infinite medium obtained from the improved solution * of the diffusion equation (IDE) by Graaf and Rinzema.

* Fluence : Phys. Med. Biol. 46 (2001) 3043-3050, Reindert Graaff and Kees Rinzema,

* "Practical improvements on photon diffusion theory: application to isotropic scattering"

* $\quad$ It is a solution for isotropic scattering

* The fluence calculated is Eq. (14) of the above reference

* For the case of low absorption, i.e., mua $<1 \mathrm{~d}-5 \mathrm{~mm} \wedge$ - 1 the coeffcient $\mathrm{k} 0$

* is approximated to MUEFF. This approximation has negligible effects

* for values of scattering typical of biological tissues.

* Note that the following arguments: s, AA, n0, and t are not used.

\section{IMPLICIT NONE}

REAL*8 mua,musp,s,t,xs,ys,zs,xr,yr,zr,n0,AA

$\mathrm{REAL} * 8$ fluence

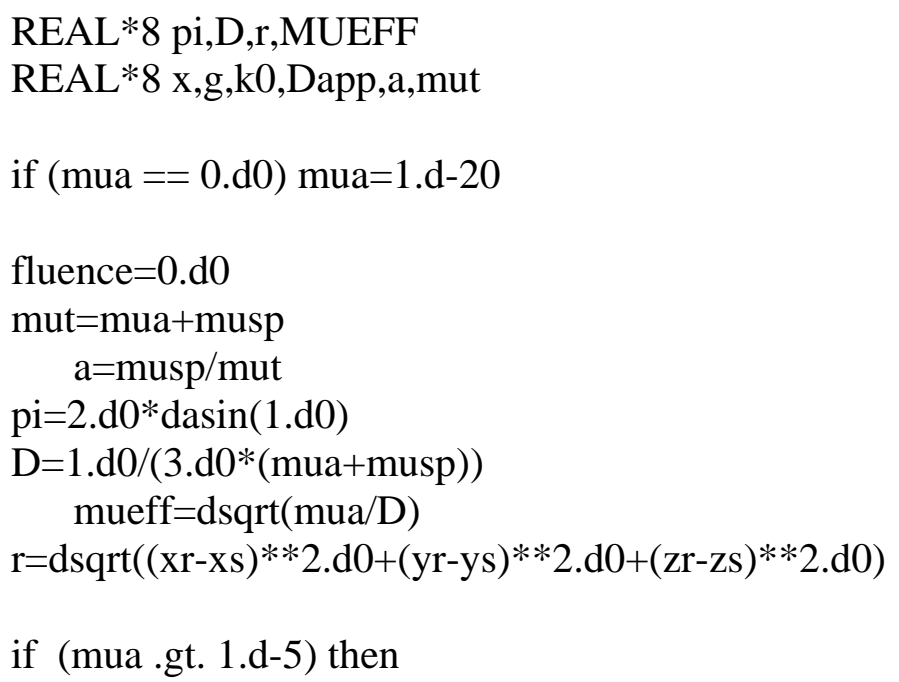




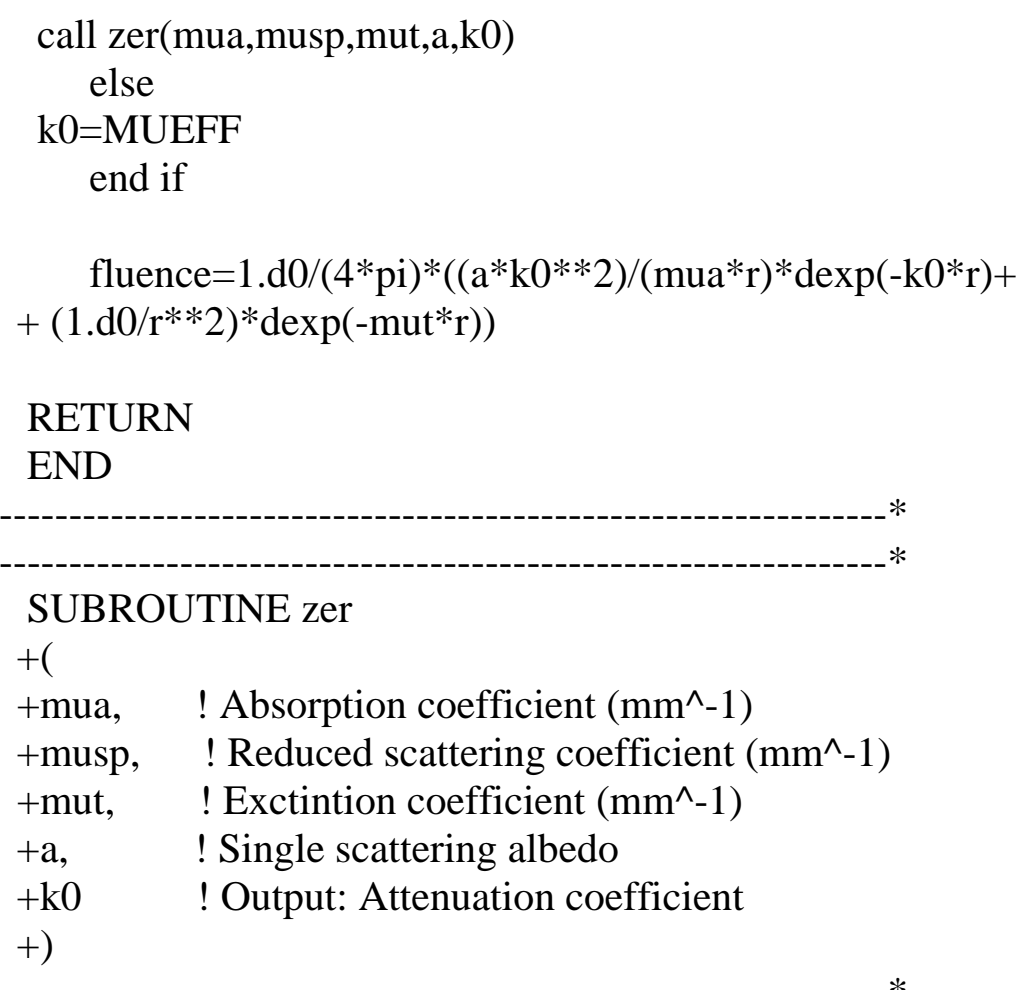

\section{IMPLICIT NONE}

EXTERNAL fun

REAL*8 a,mut,mua,musp,mueff

REAL*8 pi,D,xacc,k0,x1,x2

$\mathrm{REAL} * 8 \mathrm{fi}$

INTEGER i,j,k

$\mathrm{pi}=2 . \mathrm{d} 0 *$ dasin $(1 . \mathrm{d} 0)$

$\mathrm{D}=1 . \mathrm{d} 0 /(3 . \mathrm{d} 0 *$ (mua + musp) $)$

mueff $=\operatorname{dsqrt}(\mathrm{mua} / \mathrm{D})$

$\mathrm{x} 1=$ mueff $/ 2 . \mathrm{d} 0$

$\mathrm{x} 2=1.5 \mathrm{~d} 0 *$ mueff

xacc $=(1 . d-13)$

$\mathrm{k} 0=\mathrm{fi}$ (fun,a,mut, $\mathrm{x} 1, \mathrm{x} 2, \mathrm{xacc})$

\section{RETURN}

END

SUBROUTINE fun

$+($

$+\mathrm{x}$,

$+\mathrm{a}$,

+ mut,

$+\mathrm{fn}$,

$+\mathrm{df}$

+ ) 
IMPLICIT NONE

REAL*8 x,a,mut

REAL*8 df,fn

$\mathrm{fn}=(\mathrm{d} \log ((1 . \mathrm{d} 0+\mathrm{x} / \mathrm{mut}) /(1 . \mathrm{d} 0-\mathrm{x} / \mathrm{mut})))-(2 . \mathrm{d} 0 * \mathrm{x} / \mathrm{mut}) / \mathrm{a}$

$\mathrm{df}=-(2 . \mathrm{d} 0 / \mathrm{mut}) / \mathrm{a}+((1 . \mathrm{d} 0-\mathrm{x} / \mathrm{mut}) /(1 . \mathrm{d} 0+\mathrm{x} / \mathrm{mut})) *\left(1 . \mathrm{d} 0 /\left(\mathrm{mut}^{*}\right.\right.$

$+(1 . \mathrm{d} 0-\mathrm{x} / \mathrm{mut}))+(1 . \mathrm{d} 0+\mathrm{x} / \mathrm{mut}) /(1 . \mathrm{d} 0-\mathrm{x} / \mathrm{mut}) * * 2 *(1 . \mathrm{d} 0 / \mathrm{mut}))$

\section{RETURN}

END

FUNCTION fi

$+($

+ fun,

$+\mathrm{a}$,

+mut,

$+\mathrm{x} 1$,

$+\mathrm{x} 2$,

$+\mathrm{xacc}$

$+)$

IMPLICIT NONE

EXTERNAL fun

INTEGER, PARAMETER :: MAX=2000

REAL*8 x1,x2,xacc

$\mathrm{REAL} * 8 \mathrm{fi}$

INTEGER $\mathrm{j}$

REAL*8 df,dx,dxold,f,fh,fl,temp,xh,xl,a,mut

call fun(x1,a,mut,fl,df)

call fun(x2,a,mut,fh,df)

if(fl.eq.0.d0)then

$\mathrm{fi}=\mathrm{x} 1$

return

else if(fh.eq.0.d0)then

$\mathrm{fi}=\mathrm{x} 2$

return

else if(fl.lt.0.d0)then

$\mathrm{xl}=\mathrm{x} 1$

$\mathrm{xh}=\mathrm{x} 2$

else

$\mathrm{xh}=\mathrm{x} 1$ 


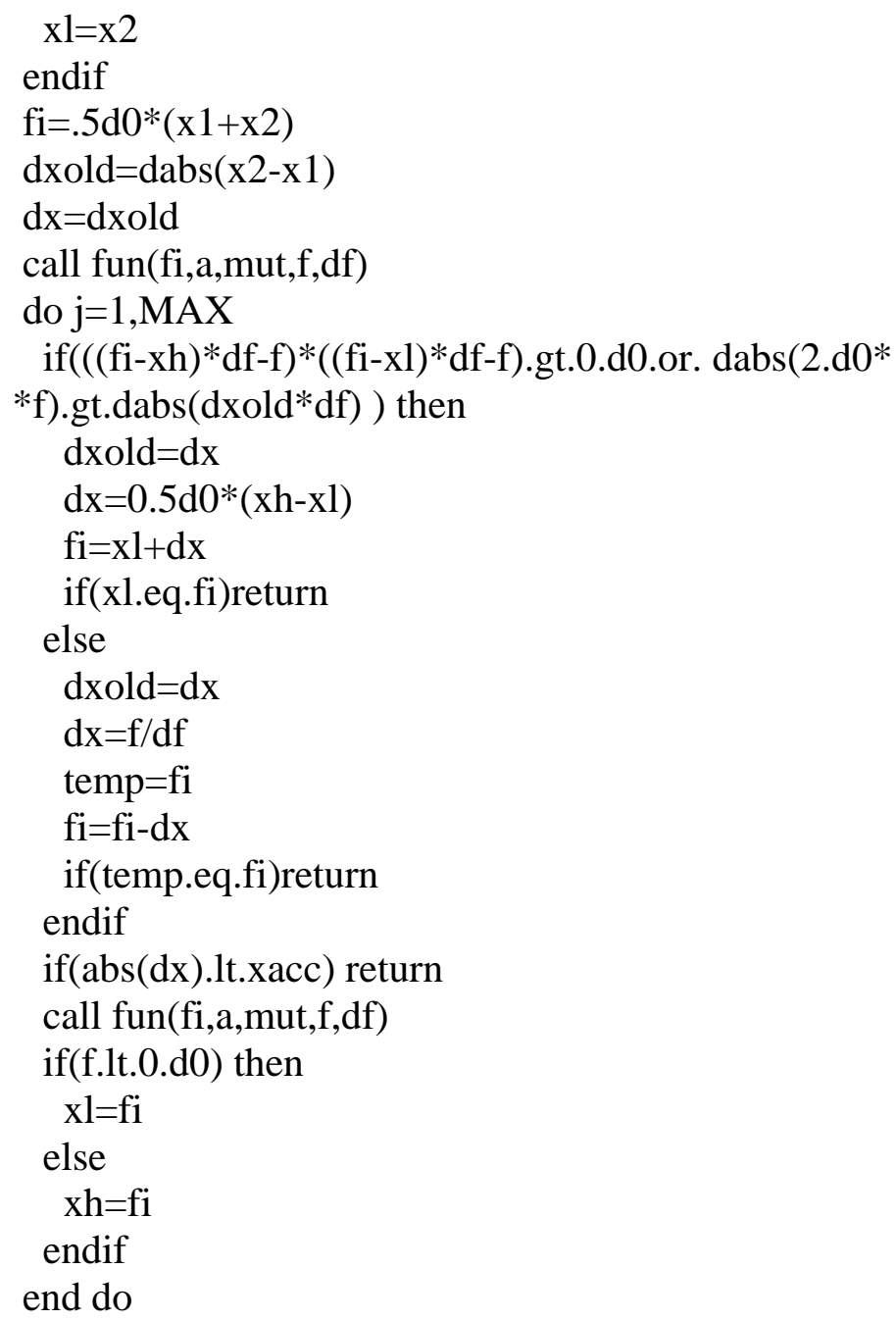

RETURN

END 